\title{
Boulance, érosion interne, renard. \\ Les instabilités sous écoulement
}

\section{A. MONNET}

Soletanche Bachy, 6, rue Watford, 92000 Nanterre

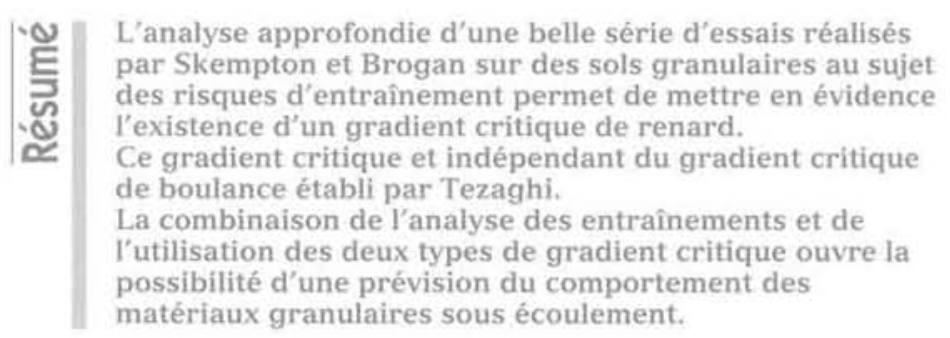
-Q L'analyse approfondie d'une belle série d'essais réalisés par Skempton et Brogan sur des sols granulaires au sujet riscues d'entrainement permet de mettre en évidence lexistence d'un gradient critique de renard. de boulance établi par Tezaghi.
La combinaison de l'analyse des entrainements et de possibilité d'une prévision du comportement des riaux granulaires sous écoulement.

\section{Boiling, washing out, piping. Internal stability under water flow}

A carefull analysis of an interesting set of experiments completed by Skempton et Brogan about washing out in granular soils allows to qualify a piping critical gradient. The critical piping gradient and the critical boiling gradient established by Terzaghi are not related.

Using the washing out analysis and the two critical gradients, the actual internal stability of granular soils could now be estimated. 


\section{Présentation}

La majorité des accidents sur les ouvrages géotechniques sont en rapport avec les effets de l'eau, et parmi ceux-ci, une proportion importante résulte de l'apparition de renards qui sont eux-mêmes le plus souvent la conséquence d'une érosion interne. Mais la prévision de ces phénomènes n'est pas évidente et il règne une grande confusion dans la description des mécanismes en jeu.

Une série d'essais réalisés par Skempton et Brogan (1994) avait pour objectif de vérifier la validité du critère d'érosion interne de Lau et Kenney. En introduisant dans l'analyse l'hypothèse que le renard est une localisation de l'écoulement dont l'apparition est commandée par un gradient critique, il est possible de quantifier l'apparition de la rupture dans tous les essais de Skempton et Brogan. La rupture se produit par boulance lorsque le gradient critique de Terzaghi est inférieur au gradient critique de renard, et par renard dans le cas contraire.

Il est alors possible de montrer que l'érosion interne n'est pas rupture en elle-même, mais qu'elle entraîne une chute du gradient critique de renard qui peut conduire à des ruptures pour des gradients beaucoup plus faibles que le gradient critique de Terzaghi.

La mise en évidence d'un gradient critique d'apparition du renard ouvre la perspective de pouvoir mettre au point des méthodes de dimensionnement pour assurer la sécurité des ouvrages vis-à-vis des instabilités sous écoulement.

\section{2}

\section{Gradient critique de Terzaghi et boulance}

Lorsqu'un échantillon de sol de porosité n, de masse volumique des grains $p_{c}$, est soumis à un écoulement vertical ascendant d'un fluide de masse volu-

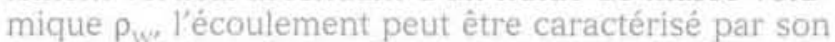
gradient, i, la variation du niveau piézométrique avec la profondeur:

$$
\mathrm{i}=\frac{\delta \mathrm{u}}{\delta \mathrm{z}}
$$

Terzaghi a montré qu'il existe une limite, le gradient critique de Terzaghi $\mathrm{i}_{\text {, }}$, au-delà de laquelle l'équilibre n'est plus assuré. Cette valeur limite du gradient résulte des conditions d'équilibre général. La perte de charge, $\rho_{w} \cdot g \cdot \frac{\delta u}{\delta z}$, ne peut être supérieure au poids immergé des grains $(1-n) \cdot\left(\rho_{G}-\rho_{W}\right) \cdot g$

$$
i_{C T}=(1-n) \cdot\left(\frac{\rho_{G}}{\rho_{W}}-1\right)
$$

Pour les sols courants, la valeur du gradient critique de Terzaghi est voisine de 1.

S'il n'existe pas d'effet de bord, la rupture se produira pour le gradient critique de Terzaghi, que le sol soit cohérent ou pulvérulent.

\section{La boulance}

Le terme de boulance est consacré pour qualifier la rupture du type analysé par Terzaghi, dans les sols pulvérulents, lorsque le phénomène se place à la surface, à l'émergence de l'écoulement. La boulance est un phénomène d'instabilité générale. Sous l'influence de l'écoulement, le sol se comporterait comme un liquide.

Les lois générales de l'équilibre ne peuvent être violées, et, dans les mêmes circonstances, dans un sol cohérent, il se produira également une rupture. Mais l'expérience montre que ses manifestations sont beaucoup plus anodines : dans cette situation, dans la pratique, on constate que la pression interstitielle est partout identique à la pression totale, sans autre manifestation immédiate. Il est possible qu'à terme cette situation conduise à une perte de cohésion, mais il n'est pas évident que cela soit toujours le cas.

\section{2}

\section{L'écoulement dans un massif}

Si l'on passe du domaine de l'échantillon à celui du massif de sol granulaire, l'existence d'un gradient local supérieur au gradient critique de Terzaghi n'est pas interdit, à condition que la zone où ce gradient est dépassé reste confinée par des terrains stables. C'est le cas des radiers injectés qui restent stables sous des gradients de 3 à 5 , pourvu qu'ils soient globalement en équilibre.

Il est possible d'exprimer le critère de stabilité de Terzaghi sous la forme plus générale: la valeur maximale de la pression interstitielle est égale en tout point d'un massif à la contrainte verticale totale. Sous cette forme, le critère est en accord avec le cas des radiers injectês.

\section{3}

\section{Les hypothèses implicites du modèle de Terzaghi}

L'analyse de Terzaghi suppose implicitement une homogénéité des forces appliquées à toutes les échelles. En réalité, pour les sols granulaires, quand l'analyse descend à l'échelle de dimension du grain, ce n'est pas le cas : les forces stabilisatrices qui résultent de la gravité s'appliquent au volume du grain, et les forces déstabilisatrices qui résultent de l'écoulement s'appliquent à sa surface.

En simplifiant, il est possible de dire que, pour un grain donné, l'effet de la gravité varie comme le cube du diamètre et l'effet de l'écoulement comme ce dernier. Sous un gradient donné, en l'absence d'autre force, la stabilité des grains fins est plus faible que celle des gros. Pour retrouver l'équilibre global, les grains fins doivent pouvoir s'appuyer sur les plus gros.

Or, cette condition n'est pas toujours assurée. 


\section{L'érosion interne et les essais de Skempton}

Dans le cas où les grains les plus fins ne peuvent pas s'appuyer sur la matrice, l'écoulement peut les entraîner.

Au point de vue de la stabilité, de nombreux auteurs ont cherché à dégager des règles et des lois de comportement permettant de maîtriser le problème des entraînements. Schuler a réalisé une revue des différentes publications sur le sujet. En particulier, l'idée qui revient à Lau et Kenney d'appliquer une condition de filtre aux gros grains par rapport aux grains plus fins, permet de définir un critère de mesure de la résistance aux entraînements sous écoulement : sont réputés stables les matériaux pour lesquels le rapport du poids $\mathrm{H}$ passant au tamis entre les diamètres $\mathrm{d}$ et $4 \mathrm{~d}$, divisé par le poids F passant le tamis de diamètre d est supérieur à 1,0 (1,3 pour les sols lâches).

Pour vérifier la validité du critère de Kenney, Skempton et Brogan ont réalisé une série d'essais de laboratoire dont les données principales sont rappelées ici.

\section{1}

\section{Les essais de Skempton}

Skempton et Brogan ont soumis à des gradients verticaux croissants une série de matériaux granulaires. Cinq matériaux de base ont été testés et utilisés pour fabriquer quatre mélanges artificiels.
La granulométrie des échantillons essayés par Skempton est rappelée sur la figure suivante. Les matériaux de base sont figurés en traits fins. Leurs principales caractéristiques figurent dans le tableau $\mathrm{L}$.

Les mélanges A, B, C et D, entre le gravier G et les sables S1, S2 et S3 figurent en trait épais sur le graphique et leurs caractéristiques sont données dans le tableau II.

Les valeurs de gradient critique d'essai citées dans le tableau II sont un peu supérieures à celles indiquées par Skempton dans le tableau IV de son article. En effet, dans notre cas il s'agit du gradient de rupture de l'échantillon, alors que Skempton cite le gradient pour lequel les entraînements sont généralisés à tout l'échantillon.

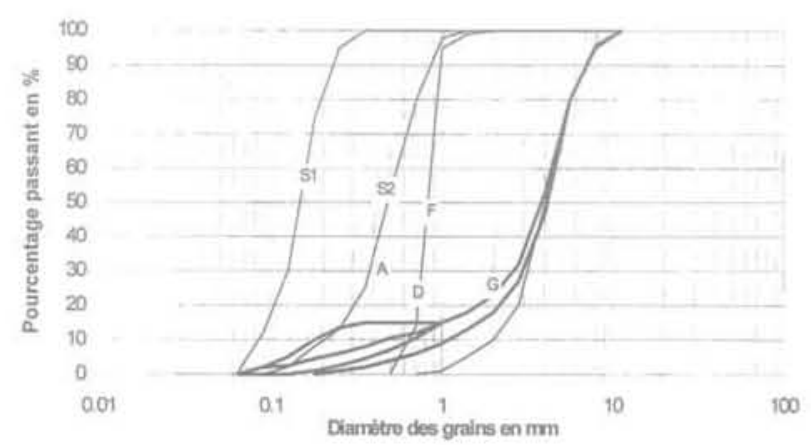

FG. 1 Granulométrie de l'ensemble des échantillons.

Grain size distribution of tested materials.

TABLEAUI Matériaux de base.

\begin{tabular}{|c|c|c|c|c|c|}
\hline & S1 & $\$ 2$ & $\$ 3$ & $\mathrm{Gr}$ & $\mathrm{F}$ \\
\hline Porosité n \% & 49 & 46 & 38 & 40 & 40 \\
\hline $\mathrm{D} 50(\mathrm{~mm})$ & 0,15 & 0,50 & 0,22 & 4,00 & 0,80 \\
\hline Perméabilité (m/s) & $1,2 \cdot 10^{-4}$ & $3,2.10^{-4}$ & $1,52.10^{-1}$ & $3.7 .10^{-2}$ & $2,5.10^{-1}$ \\
\hline Gradient critique théorique $i_{c t}$ & 0,84 & 0,89 & 1.02 & 0,99 & 0,99 \\
\hline Gradient critique d'essai $l_{c}$ & 0,69 & 0,90 & 1,01 & - & 1,05 \\
\hline Rapport $i_{C} / i_{C T}$ & 0,82 & 1.01 & 0,99 & - & 0,16 \\
\hline
\end{tabular}

TABLEAUI Caractéristiques des mélanges d'essai.

\begin{tabular}{|c|c|c|c|c|}
\hline & A & B & C & D \\
\hline Porosité n \% & 34 & 37 & 37,5 & 36,5 \\
\hline $\mathrm{D} 50(\mathrm{~mm})$ & 3,7 & 3,7 & 3,7 & 4.0 \\
\hline Perméabilité initiale (m/s) & $4,5 \cdot 10^{-3}$ & $8,5.10^{-3}$ & $8,6.10^{-5}$ & $1,8.10^{-3}$ \\
\hline Perméabilité à la rupture (m/s) & $1,8.10^{-2}$ & $2,2.10^{-2}$ & $6,2.10^{-3}$ & - \\
\hline Gradient critique théorique $i_{C T}$ & 1,09 & 1,04 & 1,03 & 1,05 \\
\hline Gradient de rupture à l'essai $i_{c}$ & 0,28 & 0,37 & 1,00 & 1,00 \\
\hline Rapport $i_{C} / i_{C t}$ & 0,25 & 0,36 & 0,97 & 0,95 \\
\hline
\end{tabular}




\section{Résultats des essais}

La figure 2 donne à titre d'exemple les éléments de l'essai sur l'échantillon A. L'indice de Kenney est donné en surimpression sur la courbe granulométrique, son échelle de valeurs est donnée sur l'axe de droite.

L'application du critère de Kenney à la courbe granulométrique de l'échantillon A montre que plus de $20 \%$ des grains, ceux dont le diamètre est inférieur à $2 \mathrm{~mm}$ peuvent être entraînés.

L'essai confirme la forte instabilité de l'échantillon. Les premiers entrainements apparaissent pour un gradient de 0,1. A partir de cette limite, la perméabilité augmente (la courbe d'essai présente une concavité tournée vers le haut). La rupture est obtenue pour un gradient d'environ 0.28 soit $25 \%$ du gradient critique de Terzaghi.

Les résultats des essais montrent que dans le cas particulier, le critère de Lau et Kenney permet effectivement de prévoir l'érosion interne. Ce mécanisme de migration des fines à travers la matrice plus grossière des échantillons a été parfaitement bien expliqué par Skempton et il n'est pas nécessaire d'y revenir ici.

Mais par contre, la prévision du gradient de rupture avancée par Skempton n'est pas convaincante.

\section{3}

\section{Rupture des échantillons}

Le poids total initial de l'échantillon A est de $4000 \mathrm{~g}$. A la fin du test, $225 \mathrm{~g}$ de matériau ont été entraînés. En admettant que le volume total de l'échantillon est resté inchangé, le volume de solide a diminué de 5,63\%. La
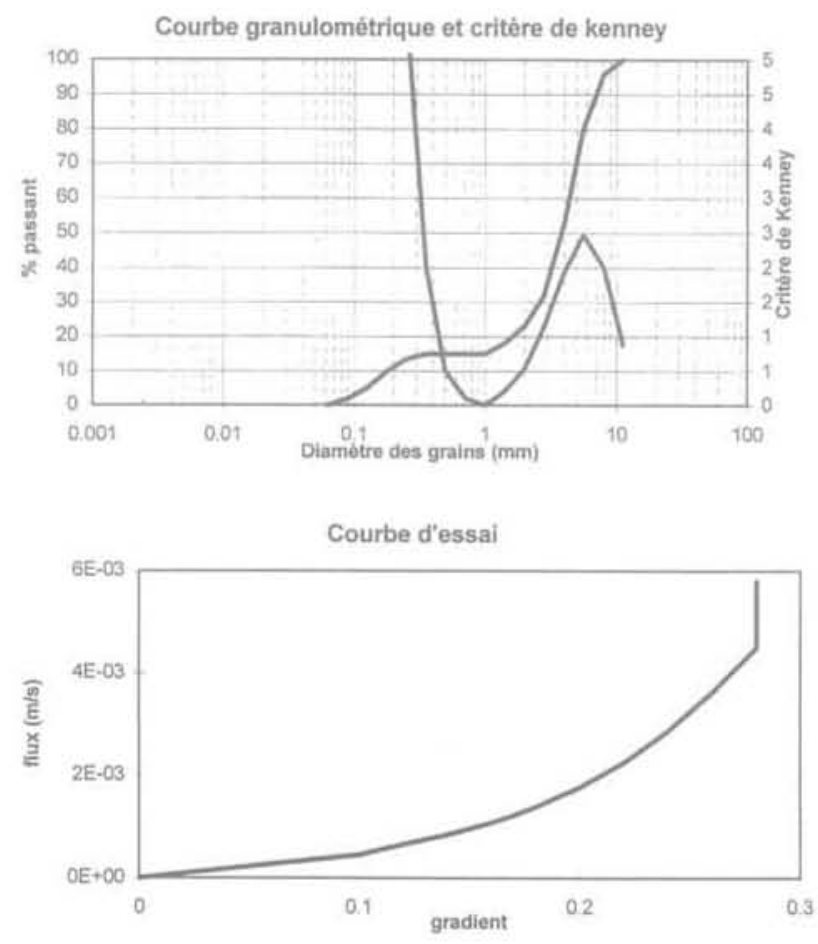

FIG. 2 Essai sur l'échantillon A. Experiment with A material. porosité du matériau est donc passée de $34 \%$ à un peu moins de $38 \%$.

Le gradient critique de Terzaghi aurait alors pour valeur $i_{C T}=1,02$ plutôt que les 1,09 de l'échantillon dans l'état initial. Avec un gradient de rupture de 0,28 on est très loin du compte.

Il est donc nécessaire d'imaginer un autre mécanisme de rupture qui ne soit pas contradictoire avec les conditions générales d'équilibre.

\section{4}

\section{Le renard, localisation des écoulements}

Notre hypothèse est ici qu'il est nécessaire que le sol présente une compacité minimale pour éviter que les hétérogénéités à petite échelle dans l'écoulement ne s'amplifient.

Dans le cas contraire, si la compacité est insuffisante, l'écoulement peut se localiser sur des lignes particulières. Si on admet que le phénomène s'amorce, il est évident que le flux va se concentrer sur ces tubes de courant localisés. Le phénomène devient alors explosif, car avec l'ouverture des « tubes » qui ne présentent pratiquement pas de frein à l'écoulement, la perméabilité globale apparente va tendre vers zéro.

Il existe quelques bons arguments qualitatifs pour supporter cette hypothèse qui n'est pas contradictoire avec les conditions générales d'équilibre:

1) Tout d'abord, il faut remarquer que des granulométries parfaitement stables au sens du critère d'entrainement de Lau et Kenney présentent des ruptures pour des gradients sensiblement plus faibles que le gradient critique de Terzaghi. C'est par exemple le cas du sable S1 qui présente une rupture pour $82 \%$ du gradient critique de Terzaghi dans les essais de Skempton. Le problème n'est donc pas obligatoirement lié à l'entrainement des fines.

2) Par ailleurs, sur la plupart des fonds de fouille arrivés en rupture sous l'effet d'un écoulement ascendant, celle-ci se manifeste par l'apparition d'un maillage de petits renards, les zones situées entre ces points restant tout à fait stables.

\section{1}

\section{Paramètres de stabilité}

A priori, nous l'avons dit plus haut, les forces stabilisatrices, $f_{1}$, sont proportionnelles au cube du diamètre des grains et les forces déstabilisatrices, $\mathrm{f}_{2}$ au diamètre. Pour un grain isolé dans un écoulement ascendant, les valeurs de ces forces seraient :

$$
\begin{aligned}
& f_{1}=\frac{1}{6} \pi \cdot d^{3} \cdot\left(\rho_{\mathrm{G}}-\rho_{W}\right) \cdot g \\
& f_{2}=d \cdot \eta \cdot v
\end{aligned}
$$

Pour le grain au sein d'un massif, la force stabilisatrice $f_{\text {}}$ conserve la même expression. On peut faire l'hypothèse que l'expression de la force déstabilisatrice va rester proportionnelle au diamètre du grain et à la vitesse moyenne de l'écoulement $v=k$.i. Le gradient limite qui commande la localisation, le gradient de 
( renard $\eta_{1} i_{p}$ serait alors une fonction du rapport de ces deux forces:

$$
i_{B}=f\left(\frac{(1-n) \cdot\left(\rho_{G} / \rho_{W}-1\right) \cdot d^{2}}{k}\right)
$$

Sous la forme la plus simple, ce pourrait être:

$$
i_{\mathrm{H}}=\mathrm{C} \cdot \frac{\mathrm{d}^{2}}{\mathrm{k}} \cdot \mathrm{i}_{\mathrm{CT}}
$$

Si ces hypothèses sont exactes, en représentant les essais dans l'espace $(\mathrm{d}, \mathrm{k})$ par le point :

- d15 (diamètre efficace de l'échantillon) ;

$$
\text { - } k=k_{\text {rupture }} \cdot i_{\mathrm{R}} / \mathrm{i}_{\mathrm{Cr}} \text {; }
$$

il devrait apparaitre une limite telle que :

- les essais pour lesquels la rupture est atteinte pour le gradient théorique de Terzaghi seraient représentés par des points situés de la limite ;

- les essais pour lesquels la rupture est atteinte pour un gradient inférieur au gradient critique de Terzaghi seraient représentés par des points situés sur la limite.

C'est ce qui semble apparaitre sur la figure 3, sur laquelle sont indiqués les points représentatifs des essais définis dans le tableau III. Les essais qui respectent la règle de Terzaghi sont notés avec les pavés vides et ceux qui la violent avec les points gras. Dans les unités retenues, millimètres pour le diamètre et mètres par seconde pour la perméabilité, la valeur du coefficient $C$ serait de 0,010 .

Il sera donc possible de prévoir le comportement sous écoulement des sols qui ne sont pas sujets aux entrainements en plaçant leur point représentatif $\left(d_{15}\right.$ k) sur le diagramme de stabilité (Fig. 4):

1) si le point représentatif se place à droite de la limite $\mathrm{k}(\mathrm{m} / \mathrm{s})=0,01 \cdot \mathrm{d}^{2}{ }_{15}(\mathrm{~mm})$ la rupture sera obtenue par boulance, pour le gradient critique de Terzaghi, $i_{c t}$ :

2) si le point représentatif se place à gauche de la límite, la rupture sera obtenue par renard lorsque le gradient atteindra la valeur $i_{R}=0,01 \cdot \frac{d_{15}^{2}}{k} \cdot i_{C r}$.

A priori, le choix du diamètre moyen $\mathrm{d}_{\mathrm{s} \text { p }}$ paraîtrait plus représentatif des forces stabilisatrices. Le diamètre $\mathrm{d}_{15}$ a été choisi ici initialement de manière opportuniste, simplement pour la raison qu'il est cité par Skempton et évite une interprétation sur des courbes imprécises. A la réflexion, c'est pourtant un meilleur choix, car il reflète à la fois la perméabilité « standard » et l'effet de la gravité.

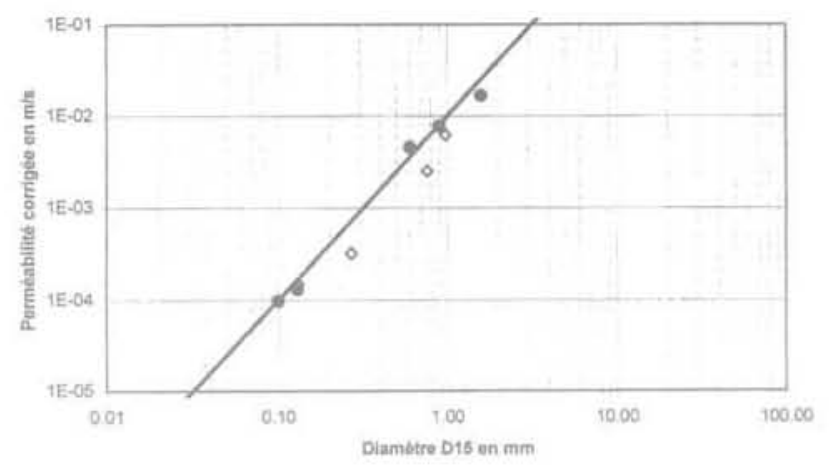

FG, 3 Valeur $\mathrm{k} . \mathrm{i}_{\mathrm{R}} / \mathrm{i}_{\mathrm{C}}$ dans les essais de Skempton.

$\mathrm{k.i} \mathrm{i}_{p} / \mathrm{i}_{\mathrm{G}}$ value in Skempton experiments.

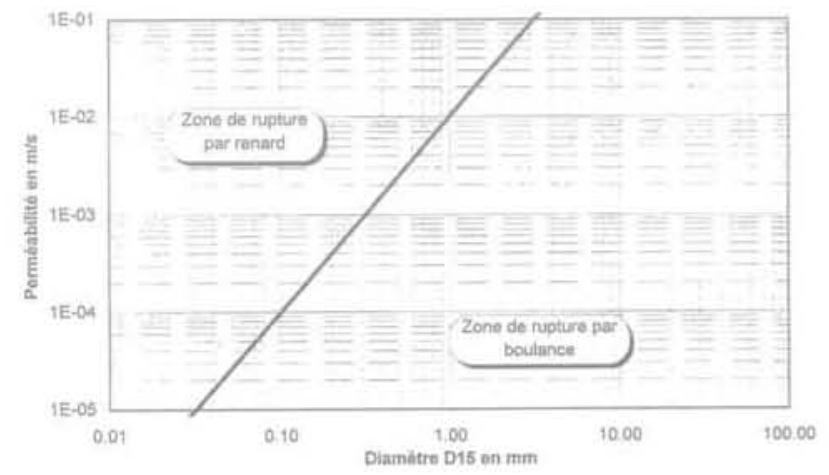

FG.4 Ligne critique de stabilité sous écoulement.

Stability chart.

Il existe des estimations de la perméabilité à partir de $\mathrm{d}_{15}\left(\mathrm{k}=0,0035 \mathrm{~d}_{15}^{2}\right.$ avec la perméabilité en $\mathrm{m} / \mathrm{s}$ et le diamètre en millimètres). Si la perméabilité est estimée par cette méthode, la comparaison avec le critère montre que tous les sols sont stables. Ce qui attire l'attention sur le fait que la perméabilité qui figure en abscisse ne peut ètre qu'une perméabilité vraie, mesurée, et non une perméabilité estimée à partír de la seule courbe granulométrique. Reste cependant ouverte la possibilité d'utiliser l'estimation de Kozeny.

L'approche utilisée ici revient au fond à utiliser la valeur de la perméabilité comme mesure de la compacité pour un sol de distribution granulométrique

\begin{tabular}{|c|c|c|c|c|c|c|c|c|}
\hline Essai & S1 & $\$ 2$ & S3 & $\mathrm{F}$ & A & B & C & D \\
\hline $\mathrm{D}_{7 \mathrm{~s}}(\mathrm{~mm})$ & 0,10 & 0.27 & 0,13 & 0,76 & 0,60 & 0,90 & 0,98 & 1,60 \\
\hline $\mathrm{K}_{\text {ruppurr }}(\mathrm{m} / \mathrm{s})$ & $1,2.10^{-4}$ & $3,2 \cdot 10^{-4}$ & $1.52 .10^{-4}$ & $2,5 \cdot 10^{-1}$ & $1,8.10^{-2}$ & $2,2 \cdot 10^{-2}$ & $6,2.10^{-3}$ & $1,8.10^{-2}$ \\
\hline$i_{H}$ & 0,69 & 0,89 & 1,01 & 1,05 & 0,28 & 0,37 & 1.03 & 1,00 \\
\hline$I_{C T}$ & 0,84 & 0,90 & 1,02 & 0,99 & 1,09 & 1,04 & 1,00 & 1,05 \\
\hline$k \cdot h / i c t$ & $9,9.10^{-5}$ & $3,2.10^{-4}$ & $1,52.10^{-4}$ & $2,5 \cdot 10^{-3}$ & $4,6.10^{-2}$ & $7,8.10^{-3}$ & $6,2.10^{-3}$ & $1.7 .10^{-2}$ \\
\hline Rupture Terzaghi & $\mathrm{N}$ & 0 & 0 & 0 & $\mathrm{~N}$ & $\mathrm{~N}$ & 0 & $\mathrm{~N}$ \\
\hline
\end{tabular}
donnée.

\section{TAaLEAUIII Données de la figure 3.}


Il existe une différence de nature entre l'expression du gradient critique de Terzaghi et celle proposée ici pour le gradient critique de renard qui est seulement une méthode d'estimation pratique d'une constante physique qui n'est pas directement accessible.

\section{2}

\section{Renard et fracturation hydraulique}

D'une certaine manière, la notion de renard envisagée ici est proche du phénomène de fracturation hydraulique.

La présentation classique de la fracturation hydraulique exprime que celle-ci apparaît lorsque la pression interstitielle imposée localement atteint la valeur de la plus petite contrainte principale. Il est généralement admis :

- qu'il s'agit d'une contrainte horizontale, la poussée au repos :

- que la valeur de la poussée au repos peut être estimée par l'expression de Jacky, $K_{0}=1-\sin \varphi$; pacité.

- que l'angle de frottement augmente avec la com-

Il est possible d'en déduire que la fracturation hydraulique se produira pour une valeur de la pression interstitielle d'autant plus faible que le matériau est compact.

Or l'interprétation des résultats des essais de Skempton et Brogan conduit à conclure, au contraire, que l'apparition du renard se produira pour une valeur de la pression interstitielle qui croît comme la compacité.

Il est vrai que les deux formes de sollicitation sont très différentes. Dans les essais de fracturation hydraulique, il s'agit de l'application ponctuelle d'une pression interstitielle, sans modification des conditions au large. Au contraire, dans les essais de Skempton et Brogan, tout l'échantillon est soumis à l'écoulement.

\section{3}

\section{Influence des entraînements}

Comme il a été dit plus haut, le critère de Lau et Kenney permet de déterminer à partir de la granulométrie si le sol est sujet aux entraînements.

Si des entraînements apparaissent, la compacité du sol va diminuer, sa perméabilité augmenter. La valeur du gradient critique de Terzaghi va diminuer légèrement, le diamètre efficace va augmenter légèrement et la perméabilité probablement beaucoup plus. Au total, la valeur du gradient critique d'apparition du renard va diminuer rapidement.

Cette estimation du gradient critique de renard à partir des résultats de l'essai $A$ et de l'expression $i_{R}=$ $0,010 . i_{C T} \cdot d_{15}^{2} / k$ est représentée sur la figure 5 . Le calcul a été fait en supposant que le diamètre efficace et le gradient critique de Terzaghi restaient constants. Le gradient critique de renard diminue rapidement et recoupe la courbe du gradient d'essai pour une valeur proche de la valeur du gradient de rupture mesuré.

Dans le cas particulier, nous disposons des valeurs d'essai pour calculer à chaque palier le gradient de renard ce qui n'est pas le cas en général.

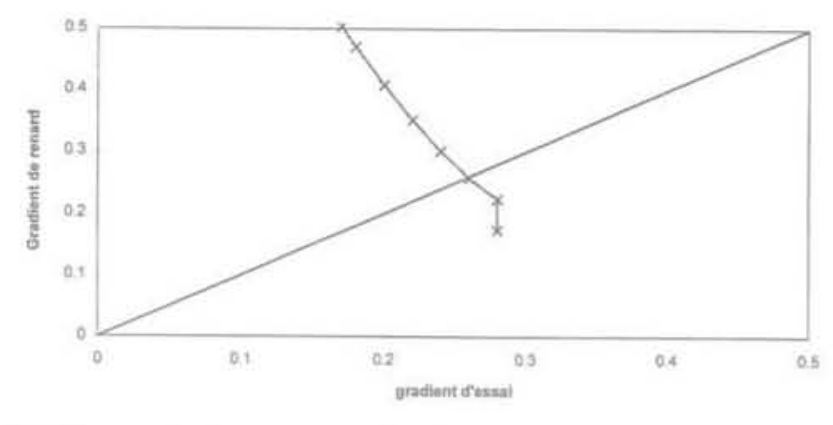

FiG.5 Détermination du gradient de renard dans l'essai A.

Piping gradient value during test $\mathrm{A}$.

On peut imaginer une méthode de prévision d'apparition du renard à partir du critère de Kenney. La mesure de la perméabilité initiale permet de caler le coefficient de l'estimation de perméabilité de Kozeny pour la granulométrie et la compacité initiales. En soustrayant à la courbe granulométrique les grains qui sont tout à la fois libres au sens de Kenney et dont le diamètre est inférieur à la limite d'entrainement de Stokes, il est possible de recalculer les nouvelles caractéristiques du sol et de déterminer le gradient de renard.

Il est tout d'abord nécessaire de faire une estimation du diamètre maximal $\mathrm{d}_{M}$ des grains qui seront entraînés pour un gradient d'essai donné.

En prenant comme mesure de la vitesse $v=k$.i pour le cas du grain dans un massif, on devrait avoir:

$$
\mathrm{d}_{M}=\mathrm{C} \cdot \sqrt{\mathrm{k} \cdot \mathrm{i} / \mathrm{i}_{\mathrm{CI}}}
$$

Pour calculer une estimation du coefficient $C$ nous ferons l'hypothèse que dans l'essai $\mathrm{A}$, la totalité du matériau qui pouvait être entraîné pour un gradient donné l'a été. Comme 5,63\% du matériau a été entraîné sous le gradient de 0,28 , pour une perméabilité de $1,8.10^{-2} \mathrm{~m} / \mathrm{s}$, ceci correspondrait à une valeur $\mathrm{d}_{\mathrm{M}}=$ $0,13 \mathrm{~mm}$, la valeur de C serait d'environ 3,4 :

$$
\mathrm{d}_{\mathrm{M}}(\mathrm{mm})=3,4 \cdot \sqrt{\mathrm{k} \cdot \mathrm{i} / \mathrm{i}_{\mathrm{CT}}}
$$

Dans l'échantillon A, les grains les plus fins ont un diamètre de $\mathrm{d}_{0}=0,065$. Le gradient de premier entraînement devrait être :

$$
i_{0}=\left(\frac{d_{0}}{3,4}\right)^{2} \cdot \frac{i_{C T}}{k}
$$

avec les valeurs initiales pour $i_{C T}$ et $k$, soit $i_{0}=0,09$, ce qui est proche de la valeur 0,10 notée par Skempton. Cela correspond également au point à partir duquel la perméabilité commence à augmenter.

Au-dessous du gradient $i_{0}$, les caractéristiques du sol restent constantes, mais au-delà toutes doivent être recalculées pour le gradient d'essai. La variation de la porosité est immédiate. Pour la perméabilité, elle peut être recalculée en utilisant l'estimation de Kozeny :

$$
\begin{aligned}
& \mathrm{k}=\mathrm{C} \cdot \frac{\mathrm{n}^{3}}{(1-\mathrm{n})^{2}} \cdot \frac{1}{\mathrm{~S}^{2}} \\
& \mathrm{~S}=6 \cdot \sum \frac{\mathrm{p}_{i}}{\sqrt{\mathrm{d}_{i} \cdot \mathrm{d}_{i+1}}}
\end{aligned}
$$


Pour l'échantillon A, la valeur de C serait environ 3. En utilisant cette valeur de la constante pour recalculer la perméabilité de l'échantillon en supprimant les $5 \%$ de grains les plus fins, la perméabilité passe à $6.10^{-2}$ $\mathrm{m} / \mathrm{s}$, ce qui est plus important que la perméabilité de $1,8 \cdot 10^{-2}$ observée à la rupture.

En fait, le temps de circulation sous gradient constant n'est pas précisé par Skempton. A chaque palier d'essai, la totalité des entraînements possibles se sont-ils réalisés? La limite de ce qu'il est possible de faire ressortir des essais de Skempton est atteinte ici.

\section{4}

\section{Influence de la turbulence}

Dans ce type d'analyse, it sera nécessaire de prendre en compte le passage à l'écoulement turbulent, qui, en entraînant une diminution de la perméabilité apparente, conduit à une amélioration de la stabilité.

\section{5}

\section{Transposition aux ouvrages}

Dans le cas des ouvrages, les situations sont beaucoup plus complexes que dans les essais de laboratoire, et aussi diverses que les types différents d'ouvrage.

Dans le cas, par exemple, d'un écoulement vers une fouille protégée par un écran, comme schématisé sur la figure 6 , quelles sont les conséquences du concept de gradient critique de renard introduit ici?

Dans le cas particulier, il est possible de distinguer trois zones où les conditions sont très différentes :

1) Dans la zone A, l'écoulement est dirigé vers le bas, la pression interstitielle est plus faible que la pression statique et la contrainte effective horizontale plus forte. L'apparition initiale d'un renard dans cette zone n'est pas vraisemblable. Par contre, si le terrain n'est pas autofiltre, les grains fins peuvent être entraînés facilement.

2) Dans la zone B, les écoulements sont plutôt horizontaux, ce qui rendrait les entraînements plus difficiles que dans la zone $\mathrm{A}$, mais la concentration des lignes de courant sous le pied de l'écran, joue en sens inverse. Les contraintes effectives qui s'opposent à l'ouverture de renards sont les charges verticales. Il est peu vraisemblable d'avoir un renard dans cette zone.

3) Dans la zone C, la situation est voisine de celle simulée dans les essais de laboratoire. La création de

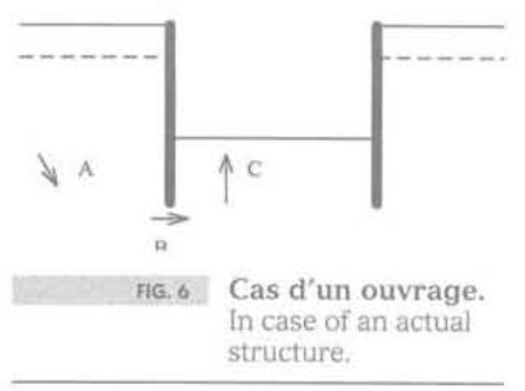

renards pourrait survenir si le gradient critique de renard est initialement inférieur au gradient critique de Terzaghi, ou le devient par érosion interne. Dans ce cas, la perméabilité apparente va chuter et les débits vont croître, avec une intensification de l'érosion interne dans les zones $\mathrm{A}$ et $\mathrm{B}$.

Finalement, ce sont probablement les conditions dans la zone C, celles que l'analyse permet de quantifier, qui commandent l'apparition d'une situation critique. Pour l'éviter, il est nécessaire que le gradient dans cette zone reste inférieur à trois limites que les méthodes, et les essais proposés plus haut permettent de définir:

- le gradient critique de Terzaghi ;

- le gradient critique de renard;

- une limite fixée par le taux d'entraînement maximal admissible,

C'est l'estimation de la distribution des gradients autour de l'écran qui pourrait être la plus grande difficulté. En effet, la plupart des sols sont hétérogènes, particulièrement les alluvions. Le rapport des perméabilités verticales et horizontales n'est pas une constante, il est fonction de la géométrie. Il sera plus important dans la zone $\mathrm{C}$ que dans les zones $\mathrm{A}$ et $\mathrm{B}$. Mais il s'agit là d'un autre sujet.

\section{6}

\section{Conclusion}

Il est certain que le critère de stabilité défini par le gradient critique de Terzaghi est insuffisant et que sa validité est limitée au cas des sols qui, tout à la fois, présentent une compacité suffisante et ne présentent pas de risque d'entraînements.

L'introduction d'une seule hypothèse supplémentaire, l'existence d'un gradient critique de renard, avec pour valeur estimée :

$$
i_{B}=0,01, \frac{d_{15}^{2}}{k} \cdot i_{C T}
$$

permet de quantifier toutes les ruptures observées dans les essais de Skempton et Brogan.

La limite d'application de l'expression du gradient critique de Terzaghi proposée ici :

$$
\mathrm{k}(\mathrm{m} / \mathrm{s})=0,10 \cdot \mathrm{d}_{15}^{2}(\mathrm{~mm})
$$

repose uniquement sur les essais de Skempton et Brogan. Des expérimentations et des analyses complémentaires sont certainement nécessaires pour conforter cette estimation et éliminer la possibilité que ces essais comportent un biais caché.

Le critère de Lau et Kenney est un bon indicateur des risques d'érosion interne et permet de détecter les sols potentiellement dangereux. Cependant, il faut remarquer que les entraînements n'impliquent pas obligatoirement une rupture. Ils peuvent cesser à un niveau acceptable. Cette propriété est utilisée par les foreurs d'eau pour « développer » les puits, ce qui le plus souvent se termine sans dégât. Pour ces sols dangereux, la méthode proposée avec l'utilisation de la formule de Kozeny sera probablement fructueuse, mais les essais disponibles actuellement sont insuffisants pour fonder une analyse. 
Dans la pratique, actuellement, en présence de sols pour lesquels le critère de Kenney détecte un risque d'entrainement, seuls des essais permettront de déterminer le gradient critique de renard. Ces essais devront être conduits par paliers de gradient, en s'assurant à chaque fin de palier que les entraînements sont terminés pour le palier considéré.

\section{Bibliographie}

Skempton A.W., Brogan J.M. - Experiments on piping in sandy gravels. Geotechnique, $n^{\circ} 3$, p. $440-460,1994$.

Shuler U. - How to deal with the problem of suffosion, Research and Development in the Fiel of Dams. CIGB-ICOLD CransMontana, Switzerland, September 7-9 1995. 